\title{
The Pressure-Speed Control of 555 Timer Circuit for Flow-Controlled Booster Motor by Solar Cell
}

\author{
Wen-Bin Lin ${ }^{1}$, Kao-Feng Yarn ${ }^{2}$ \\ ${ }^{1}$ Department of Electronic and Optoelectronic Application Engineering, ${ }^{2}$ Department of Aircraft Maintenance, \\ Far East University, Taiwan 744, ROC \\ Corresponding Author: Wen-Bin Lin
}

\begin{abstract}
This paper presents the application of 555 timer circuit. For general PWM control circuit components manner and cost reduction improvements, and apply this system of automatic flow-controlled motor. We use pressure switch to control the rpm of automatic flow-controlled motor. And then, it can enhance the water pressure due to a water tower lack. The system can reach the target of energy saving and environment friendly by using solar energy. Finally, the consumer can adjust the water flow volume by choosing low speed, middle speed, and high speed of permanent magnet DC motor revolution due to pressure switch control.
\end{abstract}

Keywords: Solar cell, 555 timer circuit, PWM control circuit, Flow-controlled booster motor, Pressure switch

\section{INTRODUCTION}

Energy is an important material basis for the survival of the current industrial and commercial society. Hundreds of years ago, the energy used by mankind mainly came from nature. However, after the Industrial Revolution, petrochemical energy sources such as coal, oil, and natural gas have risen rapidly and have gradually become the dominant energy sources in people's lives.

At present, the total annual global energy consumption has exceeded 15 billion tons of standard coal, of which about $90 \%$ is fossil energy. However, fossil energy reserves are limited and non-renewable.
If we continue to maintain the current rate of energy consumption, there may be few major fossil energy resources in the world within 50 years. At the same time, large-scale development and utilization of fossil energy have also brought serious environmental problems such as climate change and ecological destruction, which directly threaten the development of human society ${ }^{[1-5]}$.

Modern technology advances, but energy consumption has intensified, so there is the generation of alternative energy. In order to enable the sustainable development of the earth, this article implements the use of solar energy as energy to achieve the goal of saving energy and reducing pollution. With the improvement of the concept of environmental protection, the PWM control circuit is applied to the downstream pressurized motor. The design circuit is connected to the battery to provide energy to the motor to make the DC motor rotate, and then use the voltage to control the speed, and observe the changes between the motor speed and voltage.

In some bungalows with low floors or only one floor, insufficient water pressure often leads to insufficient water volume and insufficient water flow at the tap, causing a lot of inconvenience and waste of time. Therefore, we want to make an amplifier that can increase the water pressure in the water pipe, which can accelerate the water flow of the water pipe of the low-rise bungalows, increase the water flow, and make life more convenient. 

cell.

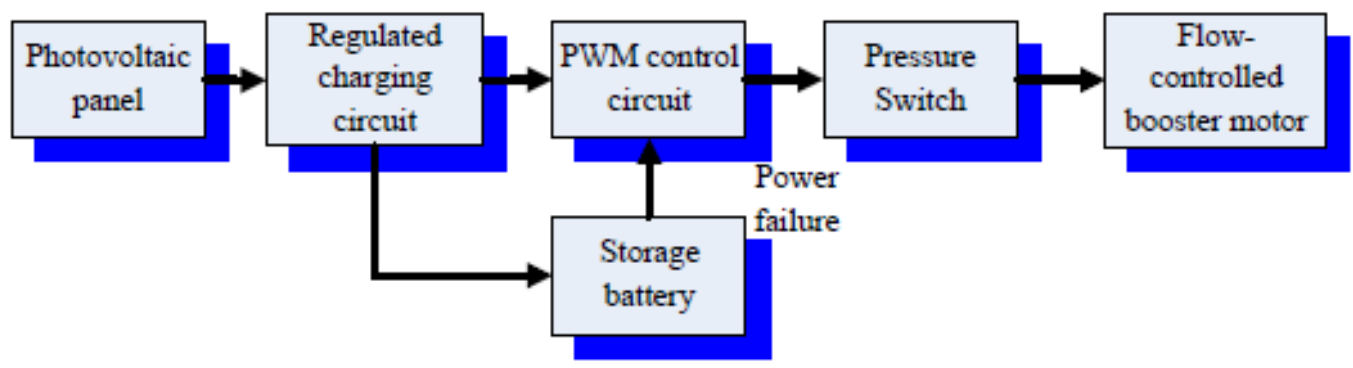

Figure 1: Block diagram of hardware composition

The Block diagram of hardware composition is shown in Figure 1. The $18 \mathrm{~V}$ Photovoltaic panel is charged to the $12 \mathrm{~V}$ battery through a voltage regulated charging circuit. The battery provides electrical energy to the PWM control circuit and (via the pressure switch) the flow-controlled booster motor.

\section{PRINCIPLE OF USING COMPONENTS}

\subsection{PWM DRIVING PRINCIPLE}

The chopper is a DC-to-DC converter. If we apply a high-speed pulse train (pulse width $<<$ time constant $\tau_{e}$ of the motor), properly control the conduction and cut-off of the transistor driving the motor, and a fixed DC power supply voltage The output voltage is converted to different levels to change the motor speed. Because the motor has inertia when it is operating, it continues to rotate during the transistor is cut off (no power consumption). Therefore, it can save power, which is more obvious in large power motors ${ }^{[11]}$.

Pulse width modulation control method is referred to as PWM control method. That is, the pulse wave period $T$ is fixed, and the conduction time $T_{O N}$ of the transistor is changed to obtain a different average output voltage $E_{A} . T_{O N}$ accounts for the proportion of the entire period $T$. Generally expressed in the duty ratio, namely

Duty ratio $D=\frac{T_{O N}}{T}=\frac{T_{O N}}{T_{O N}+T_{O F F}}$

The average output voltage $E_{A}$ can be obtained from the duty ratio,

$$
E_{A}=V_{S} \cdot \frac{T_{O N}}{T}=V_{S} \cdot D
$$

PWM (Pulse Width Modulation) is a technology that uses pulse width to adjust the output. It can be used to adjust the voltage output during used on the voltage. When the pulse is wider, the output voltage is higher; on the contrary, when the pulse is narrower, the output voltage is lower. PWM is adopted in permanent magnet DC motor speed control. Under the condition of constant frequency, changing the duty ratio to increase or decrease the overall average voltage value, so as to switch between voltage and power to save energy and control speed. In terms of control, the frequency modulation will affect the overall power and current consumption. Generally, the frequency is selected between $1 \mathrm{kHz}$ and $20 \mathrm{kHz}$. This study adopts a frequency modulation design above $20 \mathrm{kHz}$ to avoid the hearing range of normal people, so the noise generated by the motor will not be heard.

\subsection{LM555 TIMER ${ }^{[7,8]}$}

In the implementation of this paper, we are using a 555 timer as an oscillator. The oscillator forms a PWM to control the BJT switch to combine with the charging circuit in Figure 7. When the photovoltaic cell voltage is higher than the battery, the photovoltaic cell will charge the battery while supplying the motor voltage; when there is no sunlight to provide the photovoltaic cell energy, the battery will supply the 555 control circuit and motor voltage. 555 IC is a timer IC, usually used in the circuit as a monostable or unstable 

cell.

oscillation circuit, time delay, pulse generation and other functions, using an external RC circuit to make 555 generate pulse, or delay, timing, monostable state and other application circuits. The basic characteristics of 555 will be discussed in detail below ${ }^{[7,8]}$ :

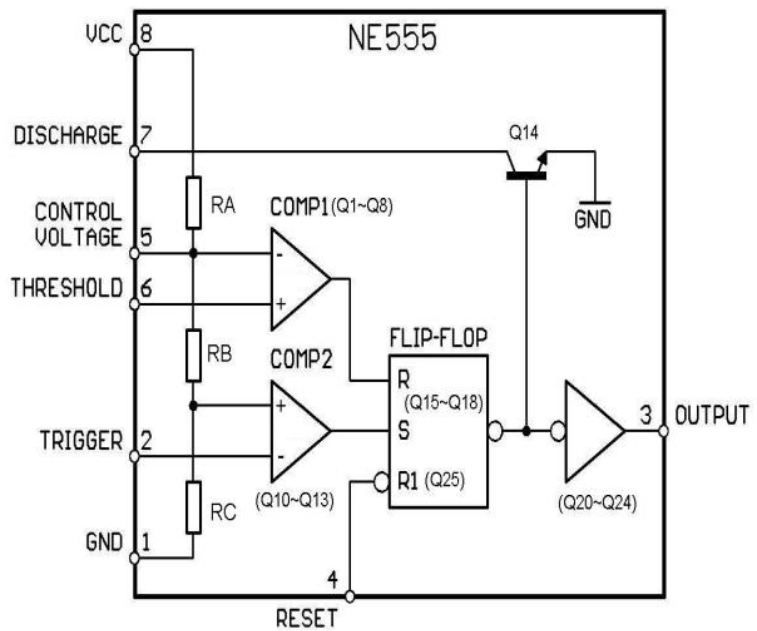

Figure 2: The internal function block diagram of the 555 timer

As shown in Figure 2, The internal function block diagram of the 555 timer. Figure 3 is the internal equivalent circuit of the 555 timer. Q1 Q8 constitute the upper comparator (COMP1), and Q10 Q13 constitute the lower comparator (COMP2). The resistor divider is composed of $\mathrm{RA}, \mathrm{RB}$, and $\mathrm{RC}$, and $\mathrm{RA}=\mathrm{RB}=\mathrm{RC}$. The reference voltage $2 / 3 \mathrm{VCC}$ supplied to the upper comparator (i.e. VCC. $(\mathrm{RA}+\mathrm{RB}) /(\mathrm{RA}+\mathrm{RB}+\mathrm{RC})=1 / 3 \mathrm{VCC}) \quad$ is connected to the base of Q13. Both comparators use Darington differential circuit. When it is used at low input current (about 100nA), a resistor that allows a wider range of time can be used. The base of Q1 (i.e. the 6th pin of IC) is used as the input terminal of the threshold. The fifth pin is the input terminal of the reference voltage of the upper comparator, which is used as a time period to add a control voltage. The base of Q10 (the second pin of the IC) is used as the trigger input.

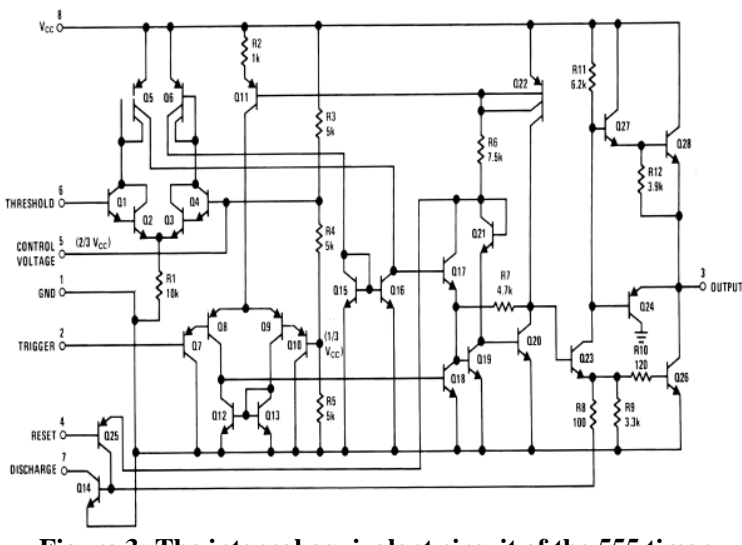

Figure 3: The internal equivalent circuit of the 555 timer

The upper comparator (COMP1) is output by the Q6 collector. The lower comparator (COMP2) is output from the Q11 collector. The above two are respectively connected to the collector and base of Q16. Q15 Q18 constitute a control Flip-Flop. Among them, Q16 and Q17 form a latch circuit.

When the base of Q10 (the second pin of IC) inputs a low potential (below $1 / 3 \mathrm{VCC})$, it will cause a positive going output of the collector of Q11. The collector of Q15 is pushed into the low potential. That is, the base of Q16 is low potential. The latch circuit accepts the set, and the collector of Q17 is low potential. The output (the third pin of the IC) is high potential, and the setting of the latch circuit continues to be maintained until it accepts reset ${ }^{[7,8]}$.

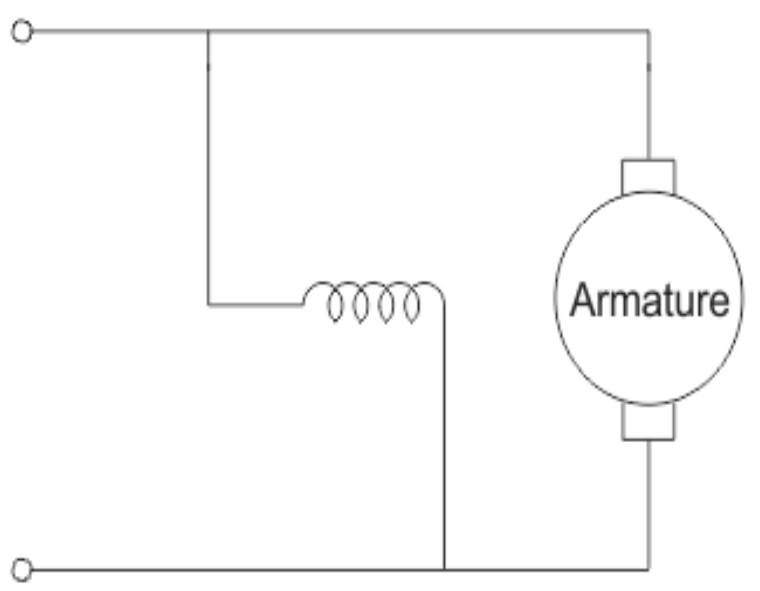

Figure 4: The equivalent circuit of the DC shunt excitation motor 

cell.

\subsection{PERMANENT MAGNET DC MOTOR}

The permanent magnet DC motor is a DC motor that uses permanent magnets as magnetic poles, and it is still a shunt excitation motor in essence. Because the structure is relatively simple, it often replaces the shunt excitation motor when driving a smaller load. The magnetic flux of a permanent magnet DC motor cannot be changed, so the speed of this motor can only be controlled by armature voltage and armature resistance. The equivalent circuit of the DC shunt excitation motor is shown in Figure $4^{[9]}$.

\subsection{OPERATION PRINCIPLE OF PRESSURE SWITCH ${ }^{[10]}$}

The unique piston/diaphragm operation principle design of the pressure switch combines the high pressure resistance of the piston technology and the precision of the diaphragm technology to make these switches work with high sensitivity. The range of repeatability is 2$5 \%$ of the highest set point. Pressure switches provide a variety of pressure switch options, from compact cylindrical type for OEM use to large packaged switch for program control.

These switches are ideal for refrigerant filtration in the machine tool industry. At the same time, it is also a reliable guarantee for the transmission of construction machinery vehicles and the redundant system as a timely monitoring of the image sensor. Figure 5 shows the crosssectional view of the pressure switch ${ }^{[10]}$.

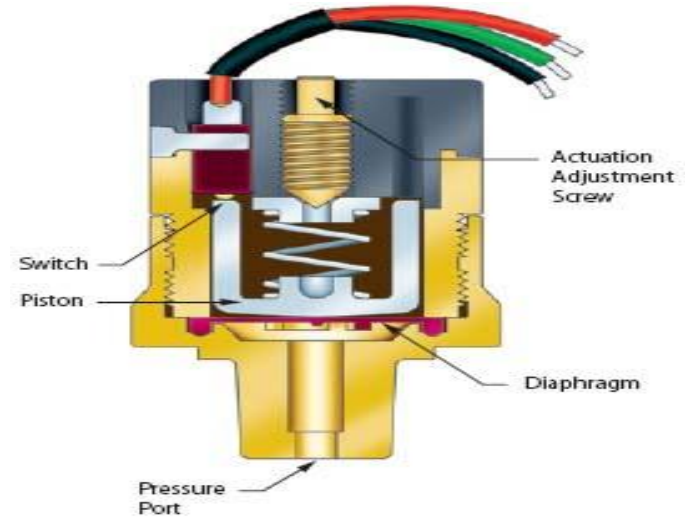

Figure 5: The cross-sectional view of the pressure switch
In this paper, the diaphragm design is adopted for the pressure switch to act on the diaphragm through the pressure guide port at the pressure connection. The diaphragm is deformed. If the pressure reaches the alarm action point of the pressure switch, the contact point will be driven after the spring is compressed. The operation of the equipment system is monitored by the on or on signal of the contact point.

\section{HARDWARE COMPOSITION}

The parts and equipment used by us are: (1) DC 12V, 30W, 3.5A, 1800RPM permanent magnet DC motor $\times 1$, (2) $8 \mathrm{~V}$, $8 \mathrm{~mA}$, solar panel $\times 4$, (3) $12 \mathrm{~V}, 12 \mathrm{Ah}$, battery $\times 1$, (4) 555 controller $\times 1$, (5) water pressure switch $\times 1$, as shown in Figure 6 .

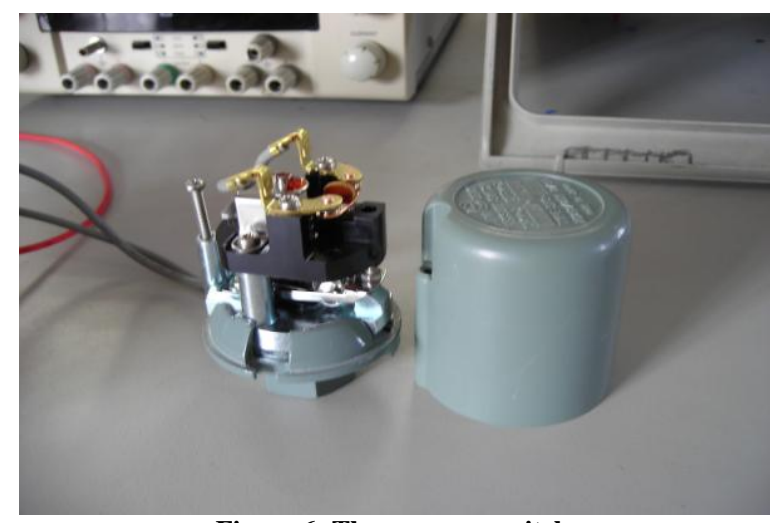

Figure 6: The pressure switch

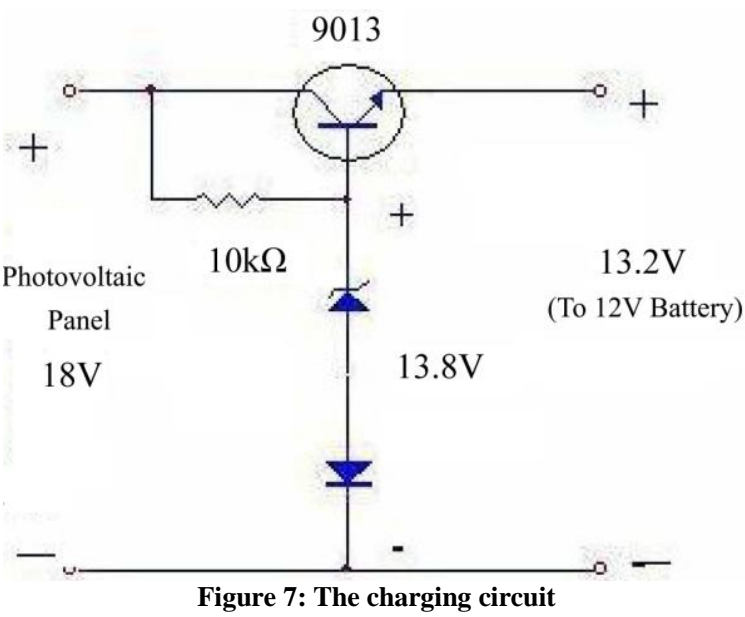

The charging circuit shows in Figure 7. The $18 \mathrm{~V}$ photovoltaic panel is switched by a protection diode and a Zener diode (guaranteed 13.8V) and 9013BJT. After 

cell.

$13.8 \mathrm{~V}$ minus the cut-in voltage $0.6 \mathrm{~V}$ of the $\mathrm{B}-\mathrm{E}$ junction of BJT, the remaining $13.2 \mathrm{~V}$ is charged to the $12 \mathrm{~V}$ battery (the battery + terminal is connected to VCC, the - terminal is connected to GND). The battery provides electrical energy to the PWM control circuit in Figure 8 and (via the pressure switch) the flow-controlled booster water pressurized motor.

The basic PWM drive control circuit shows in Figure 8. $555 \mathrm{IC}$ is connected as an unstable circuit. Silicon diodes D1 and D2 are used to control the charging and discharging path of capacitor C. Among them, the variable resistor VR is divided into the upper half $\mathrm{Ra}$ and the lower half $\mathrm{Rb}$. The operation principle is depicted in the followings. (a) Charging circuit of $\mathrm{C}$ : It is charged by R1, Ra and D2. During charging, the output is high potential, and the charging cycle is controlled by $\mathrm{R} 1, \mathrm{Ra}$ and D2. The charging cycle is Ton $=0693(\mathrm{R} 1+\mathrm{Ra}) \mathrm{C}$. (b)

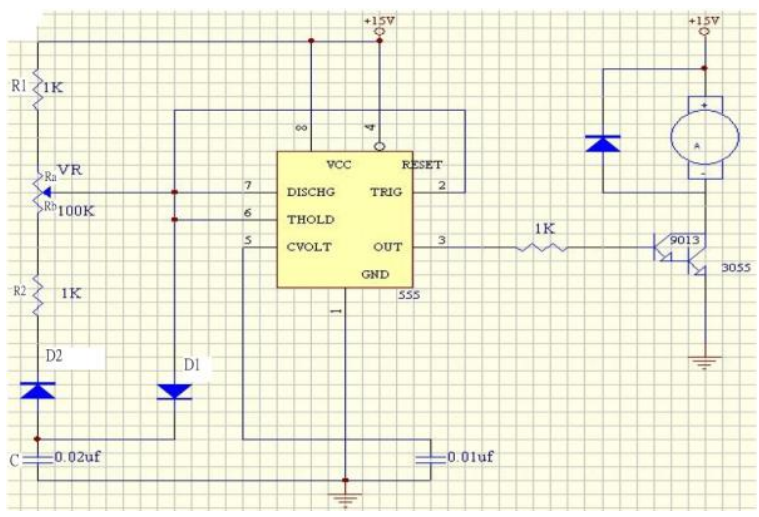

Figure 8: PWM control circuit diagram

The discharge circuit of $\mathrm{C}$ : Discharge through D1, R2 and Rb, and the output is low potential during discharge. The discharge cycle is controlled by $\mathrm{C}, \mathrm{R} 2$ and $\mathrm{Rb}$. The discharge cycle is Toff $=0693(\mathrm{R} 2+\mathrm{Rb}) \mathrm{C}^{[11-13]}$.

As long as the VR value of the variable resistance is adjusted, the ratio of charging and discharging time can be changed, and the total charging and discharging time remains unchanged. That is, the oscillation period remains unchanged. The duty ratio of the output square wave changes with VR. The DC average terminal voltage of the motor also changes in according with bandwidth modulation. The total period is $\mathrm{T}=$ Ton+Toff $=0.693(\mathrm{R} 1+\mathrm{Ra}+\mathrm{R} 2+\mathrm{Rb}) \mathrm{C} \quad[11-$ ${ }^{13]}$. When the motor is no loaded, its output waveform (pin 3 of 555IC) is shown in Figure 9.

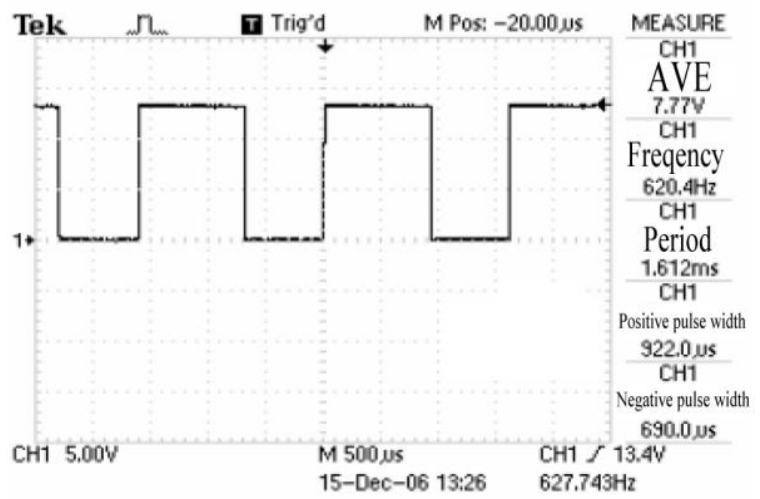

TDS 1012 - 下午 01:30:35 2006/12/15

Figure 9: Motor output waveform during no load

\section{PRACTICAL MEASUREMENT RESULTS OF PWM CONTROL CIRCUIT}

We divide three cases into actual measurement. Case 1 is low-speed operation, $12 \mathrm{~V}$ power supply, motor load consumption 0.47A, Vp-p $=8 \mathrm{~V}$. Freq $=1.4 \mathrm{~Hz}$, Duty ratio $=2.7 \%$. The output waveform of lowspeed operation is shown in Figure 10.

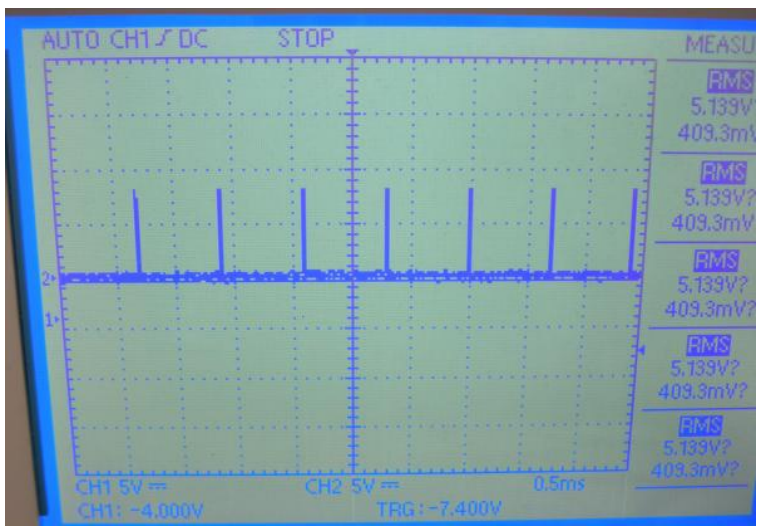

Figure 10: Motor output waveform during low-speed operation

Case 2 is running at medium speed, $12 \mathrm{~V}$ power supply, motor load consumption $0.56 \mathrm{~A}, \mathrm{Vp}-\mathrm{p}=8 \mathrm{~V}$. Freq $=1.48 \mathrm{~Hz}$, Duty ratio $=50.0 \%$. The output waveform of medium-speed operation is shown in Figure 11. 


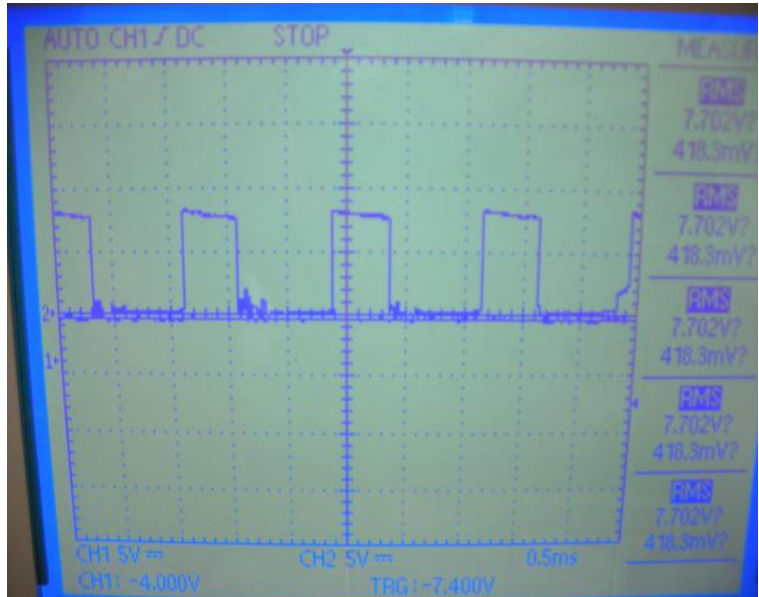

Figure 11: Motor output waveform during medium-speed operation

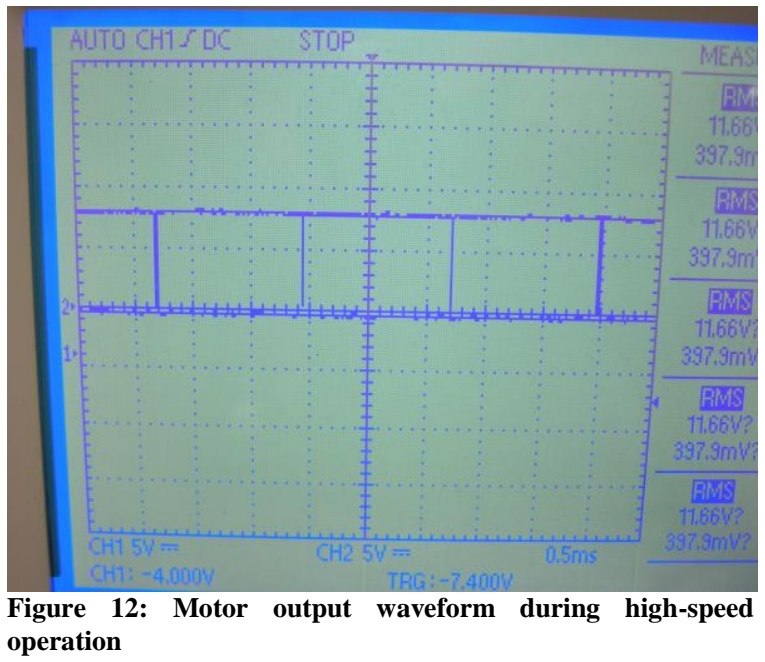

Case 3 is high-speed operation, $12 \mathrm{~V}$ power supply, motor load consumption $0.6 \mathrm{~A}, \quad \mathrm{Vp}-\mathrm{p}=8 \mathrm{~V}$. Freq $=1.58 \mathrm{~Hz}$, Duty ratio $=98 \%$. The output waveform of highspeed operation is shown in Figure 12.

\section{CONCLUSION}

Figure 13 shows the photo of the completed entity. In this paper, a 555 timer system is used to develop an application to control the speed of a flow-controlled booster motor with a pressure switch by photovoltaic energy. Since the number of components of the 555 oscillator is very small and can self-oscillate, the demand for component cost reduction is achieved. Finally, the feasibility of the system is verified through experiment.

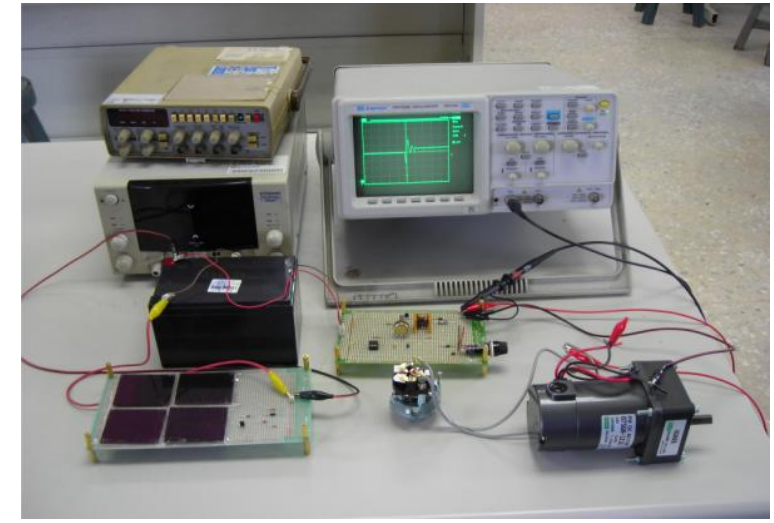

Figure 13: The photo of the completed entity

The final charging effect, that is, if the weather is good, the charging can be completed in about 12 hours. The PWM control circuit can be used to control the motor speed, which can be changed according to the demand. It is very suitable for users who have insufficient water pressure to install in the water tower. It can make life easier and not be troublesome due to small water flow.

\section{ACKNOWLEDGMENTS}

The authors would like to acknowledge $\mathrm{Mr}$. Jin-Kun $\mathrm{Wu}$ who constructed much of the hardware for the experimental system.

\section{Conflict of Interest: None}

\section{Source of Funding: None}

\section{REFERENCES}

1. Bureau of Energy, Ministry of Economic Affairs, Taiwan Energy Statistics Manual of Taiwan in 2006, Bureau of Energy, Ministry of Economic Affairs, Taipei, Taiwan, 2006. .

2. S.C. Bhattacharya, The lecture note of Renewable Energy Resources, AIT, Bangkok, Thailand, 1994.

3. J.C. Zhuang Solar Energy EngineeringSolar Cells, Quanhua Science and Technology Book Co., Ltd., Taipei, Taiwan, 2001.

4. Hinrichs and Kleinbach, Energy-Its Use and the Environment, 4th Version, Thomson Brooks/Cole, Belmont, USA, 2006.

5. C.F. Wu, Overview of Solar Power Supply System and Lighting System, Quanhua 
Wen-Bin Lin et.al. The pressure-speed control of 555 timer circuit for flow-controlled booster motor by solar cell.

Science and Technology Book Co., Ltd., pp.2-1-pp2-26, Taipei, Taiwan, 2010.

6. C.L. Huang, Development of Solar Photovoltaic Energy Grid Parallel Converter, Master's Thesis of Institute of Electrical Engineering, National Yunlin University of Science and Technology, Yunlin University of Science and Technology, Yunlin, Taiwan, 2007.

7. B.Y. Chen, Operational Amplifier Specification Sheet (2), Quanhua Science and Technology Book Co., Ltd., Taipei, Taiwan, 1992.

8. Q.X. Zheng, Sound Engineering, Quanhua Science and Technology Book Co., Ltd., Taipei, Taiwan,1997..

9. S.J. Chapman, Essentials of Electric Machine Fundamentals 4th ed, Taiwan branch of The McGraw-Hill Companies, Inc., Taipei, Taiwan, 2006,

10. http://www.kehaoauto.com.cn/article/20092-12/1007-1.htm.
11. Z.X. Lin, Motor Control Practice, Quanwei Book Co., Ltd., Taipei, Taiwan, 1999..

12. H.H. Li, and T.R.Chen, "Design and Implementation of Hartley Contactless Power Transmission System for Underwater Motor", 2010 The 9th Taiwan Power Electronics Conference and Exhibition, National Chung Cheng University, Chiayi, Taiwan. September 2010, pp.1212-1217.

13. Y.L. Ho, and T.R. Chen, "A Novel Contactless Power Transmission System Using Hartley Oscillator", The 8th Taiwan Power Electronics Conference and Exhibition, Qingyun University of Science and Technology, Zhongli, Taiwan, September 2009, pp.738-744..

How to cite this article: Wen-Bin Lin, Kao-Feng Yarn. The pressure-speed control of 555 timer circuit for flow-controlled booster motor by solar cell. International Journal of Research and Review. 2021; 8(4): 119-125. DOI: https:// doi.org/10.52403/ijrr.20210417 